\section{Arachidonic acid and infant formulas}

Advance online publication 11 March 2015. doi:10.1038/pr.2015.35

To the Editor: Interest in the relationship between long chain polyunsaturated fatty acids and infant health and development began over $40 \mathrm{y}$ ago (1) and research aimed at clarifying the role of long chain polyunsaturated fatty acids has been most prevalent during the last two decades. Throughout this time there has been a tendency to assume that from a clinical perspective docosahexaenoic acid (DHA) is the most influential of the long chain polyunsaturated fatty acids and as a consequence investigation of the independent effect of arachidonic acid (ARA) on health outcomes has been relatively neglected. The commentary by Lauritzen et al., which considers the relationship of dietary ARA to growth and development, is therefore of interest (2). The commentary starts by considering whether a lack of preformed dietary ARA results in lower brain ARA or does the infant brain receive sufficient ARA from body stores or endogenous synthesis; however, the discussion progresses to a much more potent question on the need for infant formulas to be supplemented with ARA. This latter point has important clinical implications and needs to be considered within a broader research and policy context.

There is now a wealth of experimental data indicating that DHA and ARA have important structural and functional roles at the cellular level, especially in the cerebral, cardiovascular and immune systems. However, relating the experimental findings to clinical outcome data has proved to be challenging. Although there are many RCTs showing positive effects in visual, cognitive, and immune function, there are a number of studies that have reported no additional benefit. The interventions have predominantly been a combination of DHA and ARA as this reflects the composition of breast milk, there are only a small number of DHA alone studies, and notably there are no published RCTs where the intervention is solely ARA.

With concentrations of DHA being highest in the retina and the brain, most studies have focused on visual and cognitive outcomes. In contrast, ARA has a much wider cellular distribution and is a key component of several major metabolic pathways, including those relating to prostaglandins, prostacyclines, and leukotrienes. It is evident that health outcomes relating to ARA status are likely to be diverse, and most of these have yet to be investigated. DHA and ARA are also metabolically linked through the elongation and desaturation metabolic pathways and this adds to the complexity of disentangling the independent effects of DHA and ARA on relevant health outcomes.

With ARA being a consistent and significant component of breast milk (3), a proposal to deny formula fed infants from receiving ARA needs to be supported by robust scientific and clinical evidence (4). However, the commentary correctly notes that there are quality and design issues with many of the published studies, and in particular there is a lack of clinical outcome data from solely ARA interventions. The authors also acknowledge that the metabolic equilibrium between ARA and DHA may be critically important to clinical outcomes in later life. They also highlight the potential impact of genetic variability on ARA and DHA status and acknowledge that this is at an early stage of investigation. With these many gaps in our scientific knowledge of the effects of dietary ARA in early life, the authors state that the question on whether preformed ARA in infant formulas is of any importance (good or bad) is still unanswered however they conclude with a hypothesis that dietary ARA may play a minor role on growth and development relative to that of dietary DHA.

Interestingly, this commentary coincides with the European Food Standards Authority publishing a draft Scientific Opinion on infant formula composition that states "there is no necessity to set a specific minimum content of ARA or EPA in infant formula or follow-on-formula or a specific ratio for DHA:ARA" (5). It is noted that the authors of the Lauritzen commentary are members of the Working Group on Dietetic Products that contributed to the preparatory work on the European Food Standards Authority Scientific Opinion. It is difficult to reconcile the key message in the Lauritzen commentary that it is not possible to give an evidenced based opinion on the need for preformed ARA in infant formulas with the apparent unequivocal European Food Standards Authority opinion that there is no necessity to set a minimum requirement for ARA in infant formula. The European Food Standards Authority Opinion could be enshrined in European legislation within months.

Policies need to be evidence based to ensure that the target population will experience benefit and not be at risk of iatrogenic harm. Breast milk is universally accepted as the gold standard for infant feeding and as both DHA and ARA are available to the breast fed infant, it seems sensible and equitable that, until further relevant data become available, formula fed infants should continue to receive a supply of ARA that is similar to breast fed infants. It is recognized that there are deficiencies in the quality of the current literature relating long chain polyunsaturated fatty acids to infant health and there is a need for key stakeholders to develop a more coordinated and strategic approach to future research.

Disclosure: Contributed to discussions with UK government departments, nongovernment organizations, and industry on infant nutrition policy and practice. Currently have consultancy contracts with the UK Department of Health and also DSM (Heerlen, The Netherlands), a company that produces a wide range of nutrition ingredients including docosahexaenoic acid and arachidonic acid. 


\section{Correspondence}

\author{
Stewart Forsyth ${ }^{1}$ \\ ${ }^{1}$ University of Dundee, Dundee, Scotland, UK. \\ Correspondence: Stewart Forsyth (stewartforsyth@btinternet.com)
}

\section{REFERENCES}

1. Crawford MA, Sinclair AJ. Nutritional influences in the evolution of mammalian brain. In: lipids, malnutrition \& the developing brain. Ciba Found Symp 1971;267-92.

\section{Response to Forsyth}

Advance online publication 11 March 2015. doi:10.1038/pr.2015.36

To the Editor: We are in complete agreement with Stewart Forsyth's point that, "policies need to be evidence based to ensure that the target population will experience benefit and not be at risk of iatrogenic harm." It is also correct that the commentary originated from our underlying thinking and discussions during the preparation of the recent European Food Safety Authority opinion (1). Where we disagree is on the issue of whether evidence is required in order to add arachidonic acid (AA) (or any other ingredient) to an infant formula, or whether evidence is required in order to demonstrate that AA (or any other ingredient which is present in breast milk) does not have to be added to infant formula. The former has been the norm so far. Arguing that infant formula should be similar in composition to breast milk would require the addition not only of docosahexaenoic acid (DHA) and AA, but also various immune components, as well as prebiotics and nucleotides. It would furthermore require various structural changes such as addition of the lipids as fat globules with their associated membranes as well as restructuring of the lipid molecules with palmitic acid in the sn-2 position. The European Food Safety Authority opinion does not recommend any of these things as essential either, based on the lack of evidence of their functional importancealthough many of these have been suggested to have beneficial effects and a few of them may even seem promising. The conclusion of the European Food Safety Authority paper was essentially that there was not convincing evidence that adding AA to infant formulas is necessary and hence no obligatory minimum level was suggested; however, importantly, it does not suggest that the addition of AA to infant formula should be prevented (and this also applies to several other ingredients).

The basis for our conclusion in the commentary that dietary AA is likely to be less important than dietary DHA is not only the lack of clinical evidence but also, as we point out in the first part of our paper, the fact that the AA level in the brain and other tissues is little affected by intake-whereas DHA levels are highly responsive to dietary intake of preformed DHA (2). If intake does
2. Lauritzen L, Fewtrell M, Agostoni C. Dietary arachidonic acid in perinatal nutrition: a commentary. Pediatr Res 2015;77:263-9.

3. Brenna JT, Varamini B, Jensen RG, Diersen-Schade DA, Boettcher JA, Arterburn LM. Docosahexaenoic and arachidonic acid concentrations in human breast milk worldwide. Am J Clin Nutr 2007;85:1457-64.

4. Prentice AM, Paul AA. Fat and energy needs of children in developing countries. Am J Clin Nutr 2000;72(5 Suppl):1253S-65S.

5. European Food Safety Authority. Scientific opinion on the essential composition of infant and follow-on formulae. EFSA J 2014;2:3760.

not affect status (which to some extent must be expected to serve as a pool for the generation of eicosanoids and other biologically active AA-derived molecules) and there is no evidence that addition of AA has any functional effects, then it is difficult to conclude that the addition of AA to infant formula is essential. As pointed out in our commentary, the main concern about possible adverse effects on growth of adding DHA to infant formulas without AA originated from a small trial in preterm infants (3), but none of the subsequent trials in term infants have replicated these findings. Thus, we do not consider that there is evidence to suggest that adding DHA without adding an equivalent amount of AA to formula results in adverse effects for infants.

Finally, we should also to some extent consider cost. If our aim was to produce infant formulas as similar in composition to breast milk as possible this would complicate production and thus increase the price of the product, without necessarily improving functional outcomes. We all agree that breast milk is the optimum form of nutrition in early infancy and that infant formula will always be a substitute no matter how complex; but the minimum required composition of a formula should be based on the available evidence relating to outcome rather than on the composition of breast milk per se.

\section{Lotte Lauritzen', Mary Fewtrell ${ }^{2}$ and Carlo Agostoni ${ }^{3}$}

${ }^{1}$ Department of Nutrition, Exercise and Sports, University of Copenhagen, Copenhagen, Denmark; ${ }^{2}$ Childhood Nutrition Research Centre, UCL Institute of Child Health, London, UK; ${ }^{3}$ Department of Clinical Sciences and Community Health, Pediatric Clinic, Fondazione IRCCS Cà Granda - Ospedale Maggiore Policlinico, University of Milan, Milan, Italy.

Correspondence: Lotte Lauritzen (ll@nexs.ku.dk)

\section{REFERENCES}

1. European Food Safety Authority. Scientific opinion on the essential composition of infant and follow-on formulae. EFSA J 2014;2:3760.

2. Farquharson J, Cockburn F, Patrick WA, Jamieson EC, Logan RW. Infant cerebral cortex phospholipid fatty-acid composition and diet. Lancet 1992;340:810-3.

3. Carlson SE, Werkman SH, Peeples JM, Cooke RJ, Tolley EA. Arachidonic acid status correlates with first year growth in preterm infants. Proc Natl Acad Sci USA 1993;90:1073-7. 